\title{
Collaborative Area Monitoring Using Wireless Sensor Networks with Stationary and Mobile Nodes
}

\author{
Theofanis P. Lambrou and Christos G. Panayiotou \\ KIOS Research Center for Intelligent Systems and Networks, Department of Electrical and Computer Engineering, \\ University of Cyprus, Nicosia 1678, Cyprus \\ Correspondence should be addressed to Theofanis P. Lambrou, faniseng@ucy.ac.cy
}

Received 1 August 2008; Revised 10 December 2008; Accepted 4 March 2009

Recommended by Frank Ehlers

\begin{abstract}
Monitoring a large area with stationary sensor networks requires a very large number of nodes which with current technology implies a prohibitive cost. The motivation of this work is to develop an architecture where a set of mobile sensors will collaborate with the stationary sensors in order to reliably detect and locate an event. The main idea of this collaborative architecture is that the mobile sensors should sample the areas that are least covered (monitored) by the stationary sensors. Furthermore, when stationary sensors have a "suspicion" that an event may have occurred, they report it to a mobile sensor that can move closer to the suspected area and can confirm whether the event has occurred or not. An important component of the proposed architecture is that the mobile nodes autonomously decide their path based on local information (their own beliefs and measurements as well as information collected from the stationary sensors in a neighborhood around them). We believe that this approach is appropriate in the context of wireless sensor networks since it is not feasible to have an accurate global view of the state of the environment.
\end{abstract}

Copyright (C) 2009 T. P. Lambrou and C. G. Panayiotou. This is an open access article distributed under the Creative Commons Attribution License, which permits unrestricted use, distribution, and reproduction in any medium, provided the original work is properly cited.

\section{Introduction}

Recent progress in two seemingly disparate research areas namely, distributed robotics and low power embedded systems has led to the creation of mobile sensor networks [1]. Autonomous node mobility not only brings with it its own challenges, but also alleviates some of the traditional problems associated with static sensor networks. It is envisaged that in the near future, very large scale networks consisting of both mobile and static nodes will be deployed for applications ranging from environmental monitoring to military applications [2].

In this paper we consider the problem of monitoring a large area using wireless sensor networks (WSNs) in order to detect and locate an event. In this context, we assume that the event emits a signal that is propagated in the environment. The sensors capture attenuated and noisy measurements of the signal and the objective is to reliably detect the presence of the event and estimate its position. By reliably we mean that we would like to minimize the probability of miss event (an event that remains undetected) subject to a constraint on the probability of false alarms (the sensors report an event due to noise). Note that in many applications false alarms are as bad (if not worse than) as missed events. In addition to the incurred cost for sending response personnel to the area of the event, frequent false alarms may lead the users to ignore all alarms, and as a result even detected events will go unnoticed.

To achieve reliable detection in a large area, it is necessary to deploy a huge number of sensors which with the current technology implies a prohibitive cost [3]. For example, consider a lake to be monitored for events (an event can be a boat that spills a substance in the lake that changes the water turbidity). If the lake has an area of $20 \mathrm{~km} \times 20 \mathrm{~km}$, and we assume that each sensor has a reliable sensing range (detection range) $r_{d}=10 \mathrm{~m}$, then the number of sensor nodes needed to monitor the entire lake is of the order of $10^{6}$ which with today's technology implies a prohibitive cost.

Given that it is infeasible to reliably cover the entire area with stationary nodes, in this paper we investigate an alternative way of monitoring the area using several stationary and some mobile sensor nodes that collaborate 
in order to improve the area coverage and/or to detect an event as fast as possible. The main idea is that the mobile nodes will collaborate with the stationary nodes (and with each other) in order to sample areas that are least covered by the stationary nodes. In the context of WSNs, sensor nodes are fairly inexpensive and unreliable devices, thus it is not feasible to have an accurate state of each sensor node in the field (some nodes may have failed or been carried away). As a result one cannot have all necessary information to centrally solve a path planning problem and predetermine the path that each mobile sensor node should follow in order to sample the areas least covered. In the proposed approach, mobile nodes navigate through the sensor field autonomously using only local information (i.e., the mobile node's beliefs and measurements as well as information collected from the nodes, stationary or mobile, that are in a neighborhood around the mobile).

This paper investigates the use of signal processing techniques in the path planning of mobile agents for improving the area monitoring in the context of WSNs. The main contribution of this paper is that it investigates a family of path planning algorithms and proposes a distributed algorithm that is fairly simple; it relies only on local information (i.e., information collected from the mobile's neighborhood) and can achieve very good performance. The strategy used by each mobile is based on receding horizon optimization and is motivated by the approach presented in [4] where two or more agents are moving in an area cooperatively searching for targets of interest and avoiding obstacles or threats. At every step, the mobile node tries to move toward, the least covered area, and at the same time it avoids areas covered by other nodes. In the context of WSNs, several approaches exist for identifying the point where a mobile node should go in order to improve the area coverage (for details see Section 6). All these approaches solve a static problem and to the best of our knowledge, none of them considers the path that the mobile node should follow in order to get to its destination.

The paper is organized as follows. Section 2 describes the model that has been adopted and the underlying assumptions. Section 3 presents a family of distributed path planning algorithms that can be utilized by each mobile sensor in order to navigate through the sensor field. Section 4 presents the dynamic target estimation and allocation strategy used for coverage, event detection and collaboration purposes. Section 5 presents several simulation results using various sensor fields with randomly deployed sensor nodes. Section 6 reviews related work in two research fields, the area coverage for both stationary and mobile sensor networks and the path planning algorithms in the fields of mobile robotics and unmanned aerial vehicles. The paper concludes with Section 7.

\section{Model Description and Problem Formulation}

2.1. The Environment. The environment is represented as a rectangular area $\mathcal{A}=\mathcal{R}_{x} \times \mathcal{R}_{y}$. We consider a set $\&$ with $S=|\&|$ static sensor nodes that are randomly placed in the area $\mathcal{A}$, at positions $\mathbf{x}_{i}=\left(x_{i}, y_{i}\right), i=1, \ldots, S$. In addition, we assume that a set $\mathcal{M}$ of $M=|\mathcal{M}|$ mobile sensor nodes are available and their position after the $k$ th time step is $\mathbf{x}_{i}(k)=\left(x_{i}(k), y_{i}(k)\right), i=1, \ldots, M, k=0,1, \ldots$ For notational convenience, we define the set of all sensor nodes $\mathcal{N}=\& \cup \mathcal{M}$ and reindex all mobile nodes as $m=$ $S+1, \ldots, S+M$. It is assumed that all sensors know their location through a combination of GPS and localization algorithms. Furthermore, it is assumed that all sensors can reach the fusion center (commonly referred to as sink in the WSN literature) using multihop communication.

In addition, we consider a set $\mathcal{E}$ with $E=|\mathcal{E}|$ stationary nonoverlapping event sources (sources with nonoverlapping footprints.) that are randomly placed in the environment at positions $\mathbf{e}_{j}=\left(x_{j}^{e}, y_{j}^{e}\right), j=1, \ldots, E$.

Next, we also define the neighborhood of a sensor $s$ as the set of all sensors that are located at a distance less than or equal to $r_{c}$ from the mobile. In other words, the neighborhood of sensor $s \in \mathcal{N}$ is the set of all sensors that are in the disc centered at $\mathbf{x}_{s}$ with radius $r_{c}$ :

$$
\mathscr{H}_{r_{c}}(s)=\left\{j:\left\|\mathbf{x}_{s}-\mathbf{x}_{j}\right\| \leq r_{c}, j \in \mathcal{N}, j \neq s\right\}
$$

for all $s=1, \ldots, S+M$. If $r_{c}$ is the communication range of the sensor, then $\mathscr{H}_{r_{c}}(s)$ defines all sensors that are one hop away from that node. In general however, one can define larger neighborhoods that include sensors that are two or more hops away.

2.2. Sensor Model. We assume that each event source $j \in \mathcal{E}$ emits a constant signal $V_{j}$ in the surrounding environment. As we move away from the source, the measured signal is inversely proportional to the distance from the source raised to some power $\alpha \in \mathbb{R}^{+}$which depends on the environment. As a result, the $t$ th measurement of sensor $i \in \mathcal{N}$ is given by

$$
z_{i, t}=\min \left\{V_{\text {sat }}, \sum_{j=1}^{E} \frac{V_{j}}{r_{i j}^{\alpha}}\right\}+w_{i, t},
$$

where $V_{\text {sat }}$ is the maximum measurement which can be recorded by a sensor, $r_{i j}$ is the radial distance of sensor $i$ from the event source $j$,

$$
r_{i j}=\sqrt{\left(x_{i}-x_{j}^{e}\right)^{2}+\left(y_{i}-y_{j}^{e}\right)^{2}},
$$

and $w_{i, t}$ is additive Gaussian noise with zero mean and variance $\sigma_{i}^{2}$. A sensor node reports that it has reliably detected an event if the measurement it receives is greater than the detection threshold $\tau_{d}$ (Alternatively one could use the average measurement or simply assume smaller noise variance.). This threshold is determined in a way such that the probability of false alarm is less than a given constraint $p_{f a}$. This calculation can be done as in [3] and references therein, but for the purposes of this paper, it is assumed that this threshold is given. This threshold together with $V_{j}$ defines a disc around the source (footprint of the source) where, if sensor $i$ is located inside this disc, then it will be alarmed (i.e., its measurement will be above the threshold $\tau_{d}$ ) 
with high probability, at least 0.5 . Given the model (2), the radius of the disc is given by

$$
r_{d}=\sqrt[\alpha]{\frac{V_{j}}{\tau_{d}}} .
$$

By symmetry, there exists a disc around every sensor with radius $r_{d}$ where if a source exists it will cause the sensor to be alarmed with high probability (at least 0.5 ). This is referred to as the detection (sensing) range of the sensor and it is assumed known. For the purposes of this paper, if the event occurs within this disc, then we say that it is reliably detected. Furthermore, we assume that an event is detected by the network if at least one sensor (stationary or mobile) detects the event but other fusion rules can also be used at the fusion center.

Similarly, we assume that we are given a "suspicion" threshold $\tau_{s}<\tau_{d}$ such that if the measurement of the sensor $i, \tau_{s} \leq z_{i} \leq \tau_{d}$, then sensor $i$ does not report a detection, however, it may report that it "suspects" that there may be an event around its area. Note that $\tau_{s}$ defines a disc around the sensor with radius $r_{s}>r_{d}$, and thus a node may report the suspicion if the event exists in the "donut" that is formed by the suspicion disc when the detection disc is removed. The event suspicion may be used in different ways. It can be reported to the sink which may fuse the information from several sensors or it can be given to a nearby mobile node which will collaborate with the stationary sensors in order to move closer to the suspected event area to confirm the existence or not of the event. In this paper, the suspicion will be used as in the latter example.

2.3. Objectives. The aim of this paper is to plan the path of the mobile nodes in order to achieve certain objectives. As already mentioned, the sensor network environment is constantly changing (sensors may fail or be carried away) thus it is unrealistic to expect that a central controller will have all necessary information to predetermine the paths that each mobile should follow, and thus we will consider dynamic path planning algorithms that use locally available information to determine where to go next.

In this type of problems, one can define different objectives that may result in different strategies. A possible objective is to detect and locate events as fast as possible. For this objective, a candidate strategy for the mobile nodes is to quickly move toward large uncovered areas since, if there exists an undetected event source, it is most likely located in those areas. Another possible objective is to maximize the area coverage (minimize the average probability that an event source remains undetected). In this case, a good candidate strategy for the mobile is to navigate through areas not covered by other sensors (stationary or mobile). As will be shown in the sequel, it turns out that a combination of these two strategies can achieve very good results.

To make the concept of area coverage more concrete, we divide the field area in small squares with side $d a$. In other words, we transform the sensor field area $\mathcal{A}$ into a grid $g$ of size $X \times Y$, where $X=\left\lceil R_{x} / d a\right\rceil$ and $Y=\left\lceil R_{y} / d a\right\rceil$ (see Figure 1). Thus, we assume that any sensor $s \in \mathcal{N}$ is

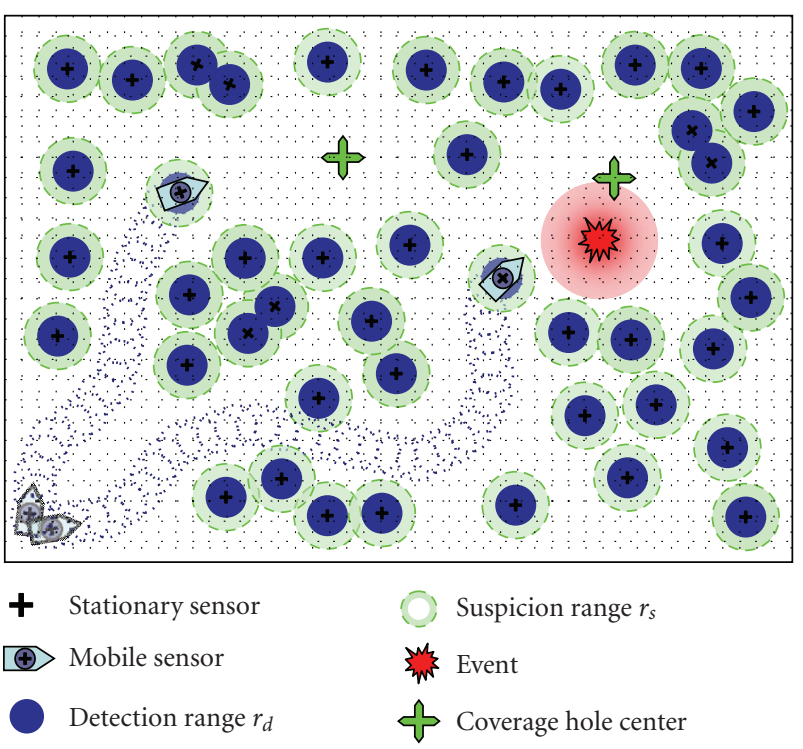

Figure 1: Environment Model.

located in the cell $\mathbf{z}_{s}=(i, j), i=\left\lceil x_{s} / d a\right\rceil$ and $j=\left\lceil y_{s} / d a\right\rceil$ (i.e., $\mathbf{z}_{s}$ is the discretized coordinate corresponding to $\mathbf{x}_{s}$ ). Furthermore, we assume that a sensor located in the cell $\mathbf{z}_{s}$, depending on the detection range $\ell_{d}=\left\lceil r_{d} / d a\right\rceil$, covers a neighborhood of cells $\mathscr{D}_{\ell_{d}}\left(\mathbf{z}_{s}\right)$ :

$$
\mathcal{D}_{\ell_{d}}\left(\mathbf{z}_{s}\right)=\left\{(p, q):(p-i)^{2}+(q-j)^{2} \leq l_{d}^{2}, \mathbf{z}_{s}=(i, j)\right\} .
$$

We associate with the grid $g$, an $X \times Y$ matrix $G_{k}, k=0,1, \ldots$, where each element of $G_{k}$ captures our "confidence" that if an event occurs in the corresponding area of the field, it will be detected by the sensor network. If the $(i, j)$ th cell falls in the detection range of a static sensor, then the corresponding $G_{k}(i, j)=1$, for all $k$ (here we use the fact that a stationary sensor may perform a long run average of its measurements and thus the probability of detecting a source in its detection range goes to 1). Otherwise, initially (at $k=0) G_{k}(i, j)=$ 0 and as the mobile nodes move around, if they sample areas not covered by the static sensors, then our confidence increases and continues to increase as the mobiles take more samples. Furthermore, if a cell has not been sampled for some time, then it is possible that our confidence will be reduced. Thus at every step, we use the following updating rule for every element of matrix $G_{k}$ :

$$
G_{k+1}(i, j)= \begin{cases}0.5 \cdot G_{k}(i, j)+0.5, & \text { if }(i, j) \in D_{\ell_{d}}\left(\mathbf{z}_{s}\right), s \in \mathcal{N}, \\ f \cdot G_{k}(i, j), & \text { otherwise, }\end{cases}
$$

where $0 \leq f \leq 1$ is the "forgetting" factor. This factor can be used to account for the physics involved with the phenomena of the events that are being monitored. For example, it can account for sources that are active only during a window of time of the observation interval or sources that turn on 
and off at various time instances. Consequently, coverage is defined as

$$
\mathcal{C}_{k}=\frac{1}{X \times Y} \times \sum_{\substack{1 \leq i \leq X \\ 1 \leq j \leq Y}} G_{k}(i, j)
$$

2.4. Mobile Sensor Node Model. The state of the ith mobile node at time $k$ is denoted by $v_{i}(k)$ which is comprised of two components, $v_{i}(k)=\left[\mathbf{x}_{i}(k), \theta_{i}(k)\right]$. As already mentioned $\mathbf{x}_{i}(k)$ is the node's position and $\theta_{i}(k)$ is its orientation (heading direction). The mobile nodes move at some constant speed $\psi$ and make path planning decisions at discrete time intervals, which means that each mobile node follows a straight line of length $\rho=\left\|\mathbf{x}_{i}(k+1)-\mathbf{x}_{i}(k)\right\|$ when moving from $\mathbf{x}_{i}(k)$ to $\mathbf{x}_{i}(k+1)$. Moreover, we point out that this model can also include maneuverability constraints of the mobile platform using some angle $\phi$ which constrains the maximum allowed difference between $\theta_{i}(k)$ and $\theta_{i}(k+1)$.

Finally, we describe the information required by each mobile in order to make path planning decisions. Each mobile uses a coverage cognitive map, an $X \times Y$ matrix $P_{k}^{m}$, $m \in \mathcal{M}$ where it keeps the state of the field. Ideally $P_{k}^{m}$ should remain $P_{k}^{m}=G_{k}$ at all times $k$, since the matrix $G_{k}$ represents the accurate global state of the field which is used for the computation of the field coverage $\mathcal{C}_{k}$. Clearly, in a dynamic environment where several sensors may accidentally move, fail or more sensors are added, it is impossible to guarantee that $P_{k}^{m}=G_{k}$ at all times. However, we emphasize, that the proposed algorithm, that will run by a mobile located at some $\mathbf{z}_{m}(k)$, computes its next position based mainly on local information, that is, information in the submatrix of $P_{k}^{m}$ that corresponds to the cells $\mathcal{D}_{\ell_{c}}\left(\mathbf{z}_{m}(k)\right)$, where $\ell_{c}=\left\lceil r_{c} / d a\right\rceil$ and thus, it is sufficient to have accurate information only for the $\mathscr{D}_{\ell_{c}}\left(\mathbf{z}_{m}(k)\right)$ submatrix. This is easily attainable since the required information can be obtained from the mobile's neighbors in $\mathscr{H}_{r_{c}}(m)$.

\section{Collaborative-Distributed Path Planning}

In this section we present a family of distributed path planning algorithms that can be utilized by each mobile sensor in order to navigate through the sensor field and to achieve its objectives. These algorithms are based on a receding-horizon approach and are motivated by [4]. In this family of algorithms, the mobile's controller evaluates the cost of moving to a finite set of candidate positions and moves to the one that minimizes the overall cost as described next. Before we proceed, to simplify the notation, in this section, we dropped the index for each mobile, that is, $\mathbf{x}(k)$ refers to the position of the $i$ th mobile sensor, $i \in \mathcal{M}$.

Suppose that during the $k$ th step, the mobile node is at position $\mathbf{x}(k)$ and it is heading to a direction $\theta$. The next candidate positions are the $\nu$ points $\mathbf{y}_{1}, \ldots, \mathbf{y}_{\nu}$ that are uniformly distributed on the arc that is $\rho$ meters away from $\mathbf{x}(k)$ and are within an angle $\theta-\phi$ and $\theta+\phi$ as shown in Figure 2. Note that the parameters $\rho$ and $\phi$ can be used to also model the maneuverability constraints of the mobile platform. At the $k$ th position, the mobile node evaluates a

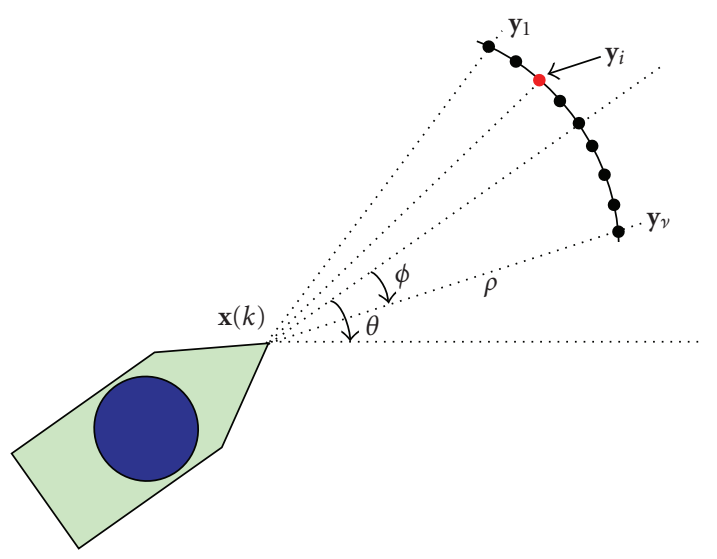

Figure 2: Evaluation of the mobile node's next step.

cost function $J\left(\mathbf{y}_{i}\right)$ for all candidate locations $\left(\mathbf{y}_{1}, \ldots, \mathbf{y}_{\nu}\right)$ and moves to the location $\mathbf{x}(k+1)=\mathbf{y}_{i}$ where $i^{*}$ is the index that minimizes $J\left(\mathbf{y}_{i}\right)$ :

$$
J\left(y_{i^{*}}\right)=\min _{1 \leq i \leq \nu}\left\{J\left(\mathbf{y}_{i}\right)\right\}
$$

The cost function $J(\cdot)$ is of the form

$$
J\left(\mathbf{y}_{i}\right)=\sum_{j \in \mathcal{F}} w_{j} J_{j}\left(\mathbf{y}_{i}\right),
$$

where $\mathcal{F}$ is a set of indeces such that the functions $J_{j}, j \in$ $\mathcal{F}$ are normalized cost functions with $0 \leq J_{j} \leq 1$ and are defined to achieve certain objectives. For the purposes of this paper, $\mathcal{F}=\{t, c, s, r, m, b\}$ but other functions can also be included. The objective of $J_{c}$ and $J_{s}$ is to achieve collaboration between the mobile and its neighboring nodes that are very close to it using only local information. On the other hand, the objective of $J_{r}$ and $J_{t}$ is to use more "global" information in order to avoid local minima. $J_{m}$ is a function for achieving collaboration between two or more mobile nodes and finally $J_{b}$ is a function for avoiding getting out of the area boundaries. Furthermore, $w_{j}, j \in \mathcal{F}$ are positive weights that tradeoff the various objectives (e.g., if it is desired that a mobile moves quickly to its target destination, then $w_{t}$ is made large).

3.1. Path Cost Functions. In this section we present the details for the cost functions that we found to give the best performance among the algorithms that we have investigated. For completeness, other functions that have been investigated are placed in an appendix.

\subsubsection{Neighboring Sensor Collaboration Cost Function Using} an Artificial Function. A main objective of the collaboration between the mobile and stationary nodes is for the mobile to avoid areas that have been covered by other nodes. The objective of this function is to push the mobile away from areas covered by other sensors. The cost function $J_{s}(\mathbf{y})$ used 
involves a repulsion force that pushes the mobile away from its closest neighbor. The form of this function is given by

$$
J_{s}(\mathbf{y})=\max _{j \in \mathcal{H}_{r_{c}}(m)}\left\{\exp \left(-\frac{\left\|\mathbf{y}-\mathbf{x}_{j}\right\|^{2}}{r_{d}^{2}}\right)\right\},
$$

where $\mathcal{H}_{r_{c}}(m)$ is the set of all nodes in the communication range of the mobile $m$. The detection range $r_{d}$ quantifies the region size around the mobile $m$ to be repelled by its neighbors. A related function that we considered consists of the total force applied to the mobile, that is, the resultant of all repulsion forces from all neighbors. However, we found that its performance was inferior to that of (10) and thus we do not consider it any further in the paper.

3.1.2. Target Cost Function. Assuming that the mobile has a target destination point $\mathbf{x}_{t}$, the cost $J_{t}(\mathbf{y})$ is a function that pulls the mobile toward its target and is a function of the distance between the mobile and the target position. This function should take a smaller value as the mobile moves toward the target destination and thus for the purposes of this paper it is given by

$$
J_{t}(\mathbf{y})=\frac{\left\|\mathbf{y}-\mathbf{x}_{t}\right\|}{\ell}
$$

where $\ell$ is the maximum distance between the mobile node and its target and is used for normalization purposes. There are several ways that one can use to assign a target position to a mobile. For example, target points may be chosen by a central controller as part of the mobile's mission. During a subsequent section we will describe alternative ways of determining the target position for each mobile. Depending on the mode of the mobile's movement, its target may be either an area that is poorly covered (monitored) or the estimated location of a "suspected" source.

All cost functions used in the paper can be easily computed by a mobile node using information in its cognitive map or by obtaining information from its neighbors. To compute $J_{t}(\cdot)$, one needs to determine a target position $\left(\mathbf{x}_{t}\right)$ and this will be done in the next section.

\section{Dynamic Target Estimation and Allocation}

In addition to the possibility of prespecifying a target position for the mobile, in this paper we investigate the possibility allowing the mobile to dynamically determine its target position $\mathbf{x}_{t}$; at every step $k$ the mobile uses the collected information to determine its new target location. We point out that it is even possible for a mobile to have two target positions, a short term as well as a longer term target (i.e., include two similar terms in (9) with different weights).

The dynamic target estimation is performed using two different algorithms depending on the state of the measurements obtained by the mobile and its neighbors as shown in Figure 3. If the mobile does not get any "suspicion" messages from its neighbors (i.e., all obtained measurements are below the suspicion threshold $\tau_{s}$ ), then the mobile is in a coverage mode and its target is the biggest coverage hole in some neighborhood around the mobile (the size and shape of this area can be a parameter of this problem). On the other hand, if the mobile receives at least a "suspicion" message then it goes into the search mode and the target becomes the estimated event source position. Finally, if an event source is detected by the mobile, we assume that it is neutralized and that the mobile moves towards its next target (This is a modeling assumption that may not be very practical. On one hand we may assume that the actual time between the step that the mobile detected the event and the next one is long enough to allow a response crew to respond. On the other hand, the mobile may be programmed to ignore (subtract) the signal from the known sources so it can continue its mission.) . Next we present the specific algorithms used in each case.

\subsection{Coverage Hole Estimation Scheme-Zoom Algorithm.} In this subsection we present a computationally efficient algorithm for coverage hole detection. Using the coverage hole detection algorithm a central controller (e.g., the sink) can estimate the coordinates of up to the $M$ biggest coverage hole centers (which can become the target coordinates of the $M$ mobiles). In other words, the aim of this algorithm is to determine where the $M$ mobiles should be placed in order to maximize coverage (i.e., maximize (7)). We emphasize that this algorithm can run either by any central controller on the entire field to obtain up to $M$ coverage holes, or by each mobile node itself, to estimate the coordinates of the biggest coverage hole center inside a neighborhood $r_{c}$ at each moving step $k$. Since this algorithm may run frequently (as new information regarding the state of the field becomes available) it is required that it is computationally efficient.

Using the principle of divide and conquer we propose the Zoom Algorithm which is very efficient in computation complexity, time and memory. The idea is to divide the grid (i.e., the matrix $G_{k}$ ) or any subgrid (i.e., a submatrix of $P_{k}^{m}$ that corresponds to the cells $\left.\mathscr{D}_{\ell_{c}}\left(\mathbf{z}_{m}(k)\right)\right)$ in four equal segments, and choose the segment with the maximum number of empty cells, that is, the segment with the maximum number of cells with $G(i, j)=0$. (Alternatively, one can choose the segment with the least coverage as defined by (7)). Then, this procedure is repeated either until the segment size is equal to a single cell or until all segments have the same number of empty cells. In the first case, the hole center position will be the center of the cell. In the second case, the hole center position will be the lower right corner of the upper left segment (the center of the segment during the previous iteration). Figure 4 illustrates the idea of zooming for hole detection when this algorithm is used by each mobile node in a distributed fashion. The details of the algorithm, when it is used by a central controller, are listed in Algorithm 1.

More information and comparative theoretical and simulation results between the zoom algorithm and other ways of finding the coverage holes can be found in [5].

4.2. Source Position Estimation Scheme. As mentioned earlier, as each mobile node $m$ navigates in the field, it continuously 


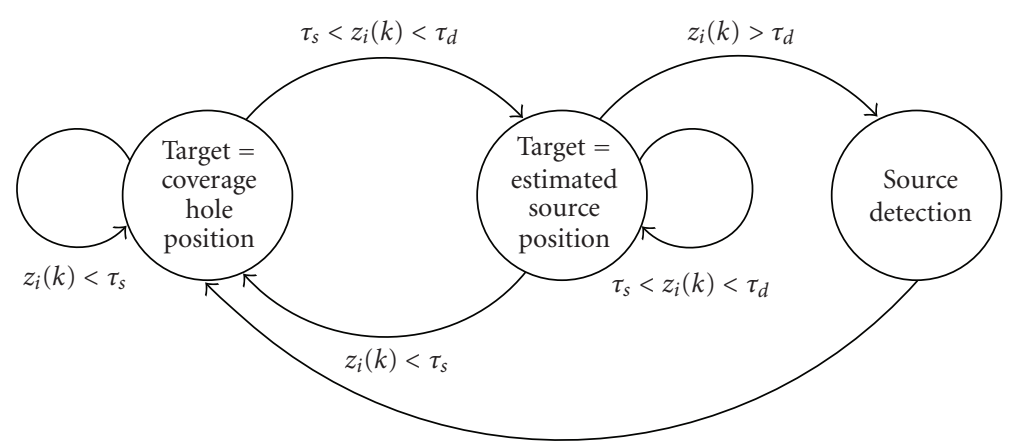

FIgURE 3: The target allocation strategy for the $i$ th mobile sensor node during the $k$ th step.

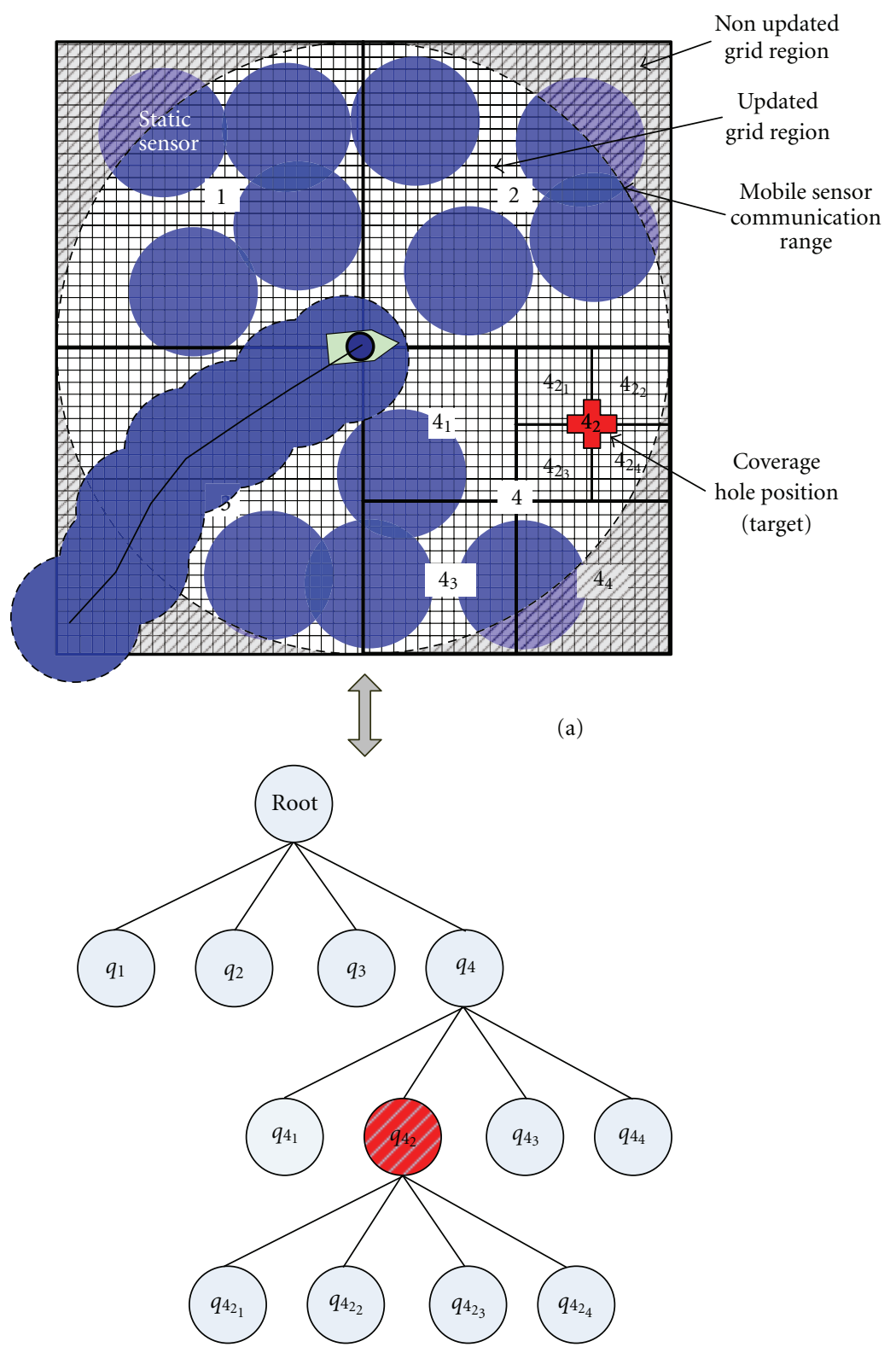

(b)

FIGURE 4: Illustration of the zoom algorithm (a) Grid segmentation (b) Generated tree. 


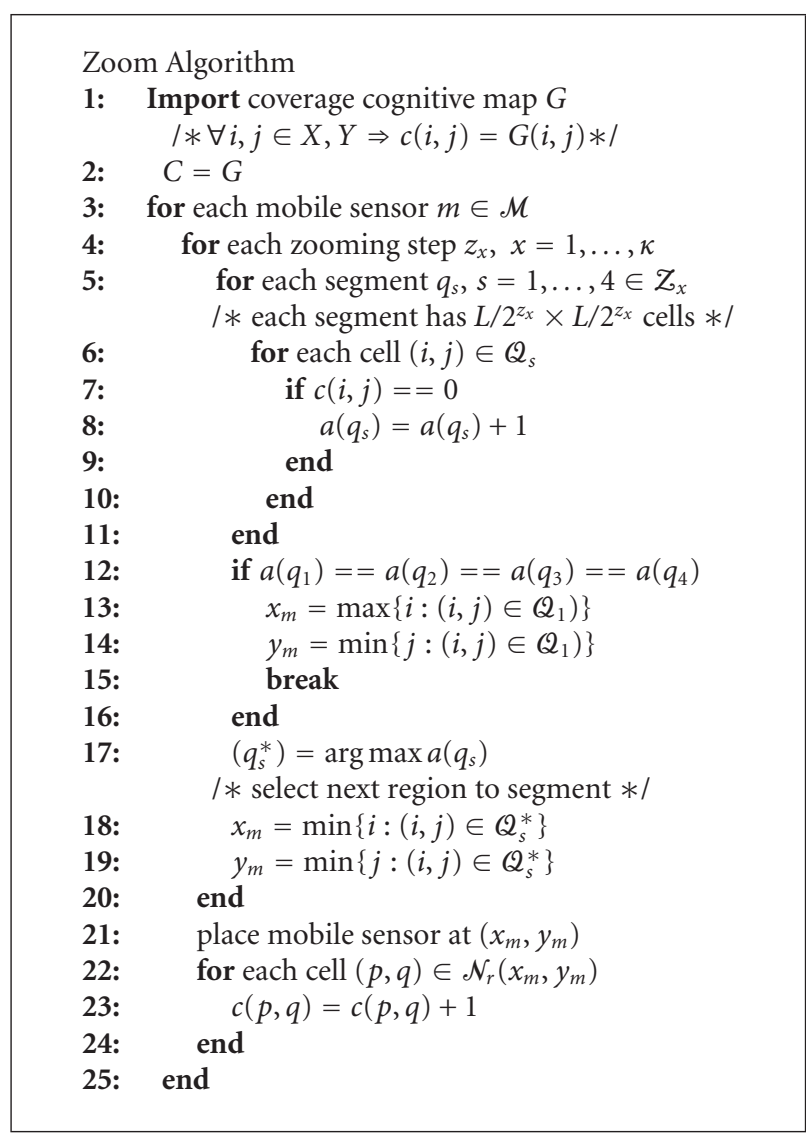

Algorithm 1: Pseudocode for the Zoom Algorithm.

samples the environment and also queries its neighboring nodes about their positions and their sensor measurements $z_{j}, j \in \mathcal{H}_{r_{c}}(m)$. In the case when one or more sensor readings are between the $\tau_{s}$ and $\tau_{d}$ thresholds, the mobile node uses the measurements to estimate a likely position of the source which will then become its target location. For this estimation, a number of estimation algorithms can be used (e.g., see [6-8]). For the purpose of this paper non linear least squares estimation has been used. The event source location (target position) $\mathbf{x}_{t}=\left(x_{t}, y_{t}\right)$ is the solution to the minimization problem:

$$
J=\sum_{i \in \Omega(k)}\left(z_{i}-\frac{V}{\left[\left(x_{t}-x_{i}\right)^{2}+\left(y_{t}-y_{i}\right)^{2}\right]^{\alpha / 2}}\right)^{2},
$$

where $\Omega(k)$ is a set of measurements that includes the measurements of the mobile's neighbors at the $k$ th step together with any measurements obtained by the mobile up until step $k$. In this paper, a uniform diffusion model [8] has been adopted and also the initial source concentration $V$ is assumed to be known. We point out however that extension for the case where $V$ is unknown is straightforward. As long as the mobile continues to get "suspicion" signals, it continues to search for the source by updating the estimated source position. As before, once the source are detected, it is assumed that it is neutralized and the mobile resumes its coverage function.

4.3. Distributed Target Allocation. The previous two subsections describe two different methods that can be used by the mobiles in order to autonomously decide their target location. Both methods utilize information that can be obtained by the mobile from its neighborhood. In the case of the coverage hole estimation, the information is included in a relevant submatrix of the cognitive map, while for the source position estimation the relevant information is the measurements of the neighboring nodes. A possible problem arises when two or more mobiles are close to each other. In this case, it is very likely that the information they will use to estimate the target position will be the same and as a result they will all estimate the same target location. Clearly, this is not a good collaboration strategy since there is no benefit if they all converge to the same point.

To avoid this problem we utilize the following two protocols depending on the state of the mobile node (i.e., searching for a source or coverage).

If a mobile node $m$ is in searching mode and also in communication range with other mobiles, then it queries its neighboring mobiles for their current position and their target locations. Then, it computes the distance between its own target and the target of the neighboring mobiles $d_{m, j}^{t}$ for all neighboring mobiles $j$,

$$
d_{m, j}^{t}=\left\|\mathbf{x}_{m}^{t}(k)-\mathbf{x}_{j}^{t}(k)\right\| .
$$

If this distance is greater than a threshold value then it assumes that the two mobiles are heading toward different targets and thus it continues normally. If $d_{m, j}^{t}$ is less than the threshold value then it is very likely that the two mobiles are heading toward the same suspected point and thus only one should continue the search toward that target while the other should switch to the coverage mode. This decision is based on the distance of each mobile from its target. The mobile that is closest to its target continues the search while the other switches to the coverage mode. For the purposes of this paper, the threshold distance used to decide whether two mobiles are heading toward the same target is set to $2 r_{d}$.

Now if a mobile node $m$ is in the coverage mode and is also in neighborhood of other mobiles, then, in order to avoid going toward the same point, it queries the other mobiles in its communication range for their current locations and their target points. Once a mobile has received the target points of all mobile neighbors, then it updates its cognitive map and assumes that these target points constitute covered areas. Then it proceeds normally with the coverage hole estimation algorithm (Zoom Algorithm). With this simple scheme, the mobiles avoid exploring the same areas. This scheme has some important benefits. It is distributed (no need for a central controller), it is simple, and it utilizes only local information (the relevant information in the submatrix $\mathscr{D}_{\ell_{c}}\left(\mathbf{z}_{m}(k)\right)$, which corresponds to the neighborhood $r_{c}$ of the cognitive map).

Finally, it is worthwhile to mention that when two mobiles come into communication range, they can also 
exchange their cognitive maps so that a mobile does not explore areas already explored by other mobile nodes.

\section{Simulation Results}

In this section we present some simulation results with some representative scenarios that show the movement of a set of mobile nodes and also compare the performance of different path planning algorithms (all from the family of algorithms presented in Section 3). Depending on which cost functions are used in (9) and the weights, one can obtain different algorithms. To distinguish between the different algorithms investigated, we use acronyms where each letter corresponds to the individual cost functions used, for example, TS refers to an algorithm for which $w_{t}>0$ and $w_{s}>0$ while $w_{c}=$ $w_{r}=w_{m}=0$. (For all algorithms and all experiments to prevent any mobile from going outside the area we have used $\left.w_{b}=1\right)$.

Unless otherwise stated, all experiments refer to a square $300 \mathrm{~m} \times 300 \mathrm{~m}$ field, and a grid with $d a=1 \mathrm{~m}$ is used. The mobile maneuverability parameters are set to $\rho=2 \mathrm{~m}$ and $\phi=30^{\circ}$ while for every decision $v=10$ candidate next positions are considered. For the event propagation model, we assume that $V=1500, V_{\text {sat }}=100$, and the exponent $\alpha=2$. Finally we assume that a detection threshold $\tau_{d}=15$, and thus the sensing radius of all sensors (stationary and mobile) is $r_{d}=10 \mathrm{~m}$ and the communication radius $r_{c}=$ $4.5 \cdot r_{d}=45 \mathrm{~m}$ (for the neighborhood of each sensor we only consider its one hop neighbors).

Next we present some representative scenarios and show the movement of a team of robots that uses the Distributed TS algorithm, a simple algorithm that performed very well against all other algorithms investigated. In this algorithm, every mobile makes autonomous decisions using only the $J_{t}$ (with $\ell=r_{c}$ ) and $J_{s}$ cost functions (i.e., $w_{t}=0.8, w_{s}=0.2$, and $w_{c}=w_{r}=w_{m}=0$ ). For estimating the target positions, the mobile uses either the coverage hole detection algorithm (in coverage mode) or the source position estimation algorithm (in search mode) and the distributed target estimation scheme presented in the previous section. Finally, for the coverage hole detection algorithm only the cells in $D_{\ell_{c}}\left(\mathbf{z}_{m}(k)\right)$ are used. In other words, the coverage hole is estimated only in its neighborhood.

In the first simulation experiment we use a team of two mobile nodes and show the behavior of the Distributed TS algorithm in a field with 100 randomly deployed stationary sensors. In this simulation scenario there is no event source thus Figure 5 shows how the two mobile nodes navigate collaboratively through the field, sampling points that are not adequately covered by the stationary sensors. As seen from the paths followed, there is collaboration between mobile and stationary sensors in the sense that the mobiles have found two different paths that are least covered by the stationary sensors. Also notice how the two mobiles collaborate and select different targets at the beginning of their motion. Moreover note that one can adjust the mobile's parameters in order achieve different objectives. For example, Figure 5(a) shows a path where the mobiles move quickly through the field to achieve faster detection. On the

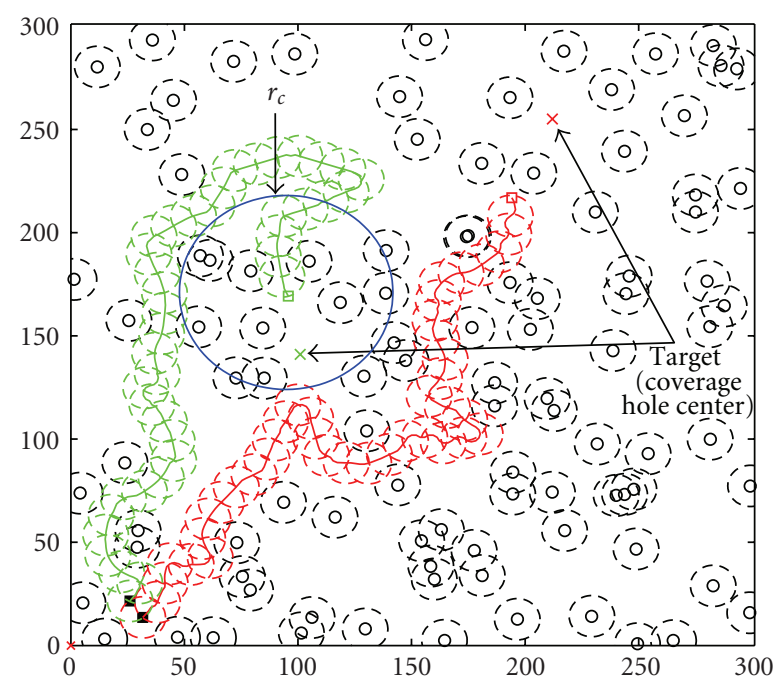

(a) Paths followed when the mobile's objective is fast detection ( $w_{t}=$ $\left.0.8, w_{s}=0.2, r_{c}=45 \mathrm{~m}\right)$

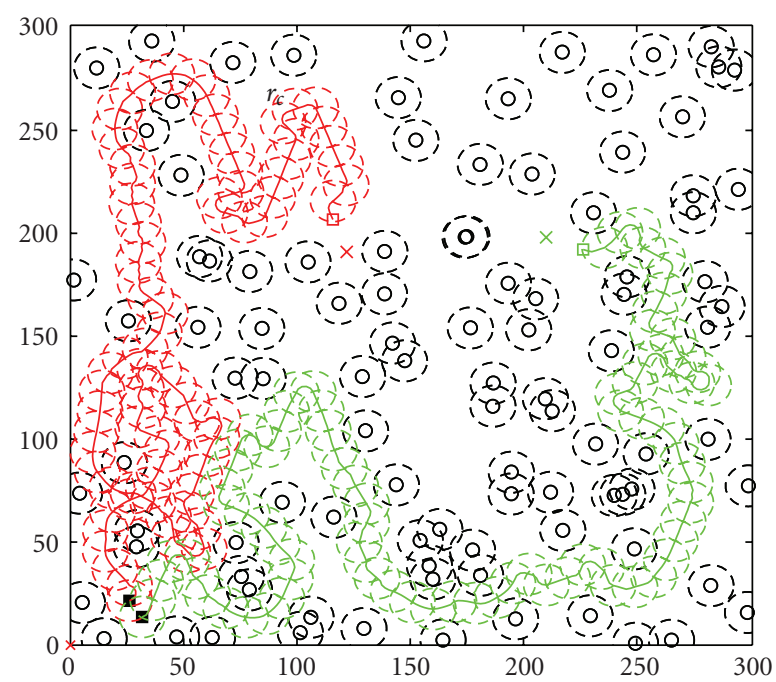

(b) Paths followed when the mobile's objective is better coverage $\left(w_{t}=0.2, w_{s}=0.8, r_{c}=25 \mathrm{~m}\right)$

FIgure 5: Dynamic path planning using a team of two mobile nodes.

other hand, Figure 5(b) shows a scenario where the mobiles try to achieve better coverage by covering a hole before they proceed. Finally, we point out that given enough time, all algorithms will cover the entire field.

Figure 6 shows the paths followed by two mobile nodes when a set of five nonoverlapping static sources exist (each source is turned on at the beginning of the simulation time and stays on for the entire simulation with $V=3000$ ). We assume 100 randomly deployed sensors in the field. The detection threshold of all sensors is $\tau_{d}=30$ (thus $r_{d}=$ $10 \mathrm{~m})$, and the suspicion threshold is $\tau_{s}=5\left(r_{s}=24.5 \mathrm{~m}\right)$. Figure 6 also shows the positions of the event sources. One source is reliably detected by the stationary sensors however for the remaining four there are no stationary sensors in a radius $r_{d}$ around the event, and thus these events would have remained undetected. Initially, both mobile nodes are 


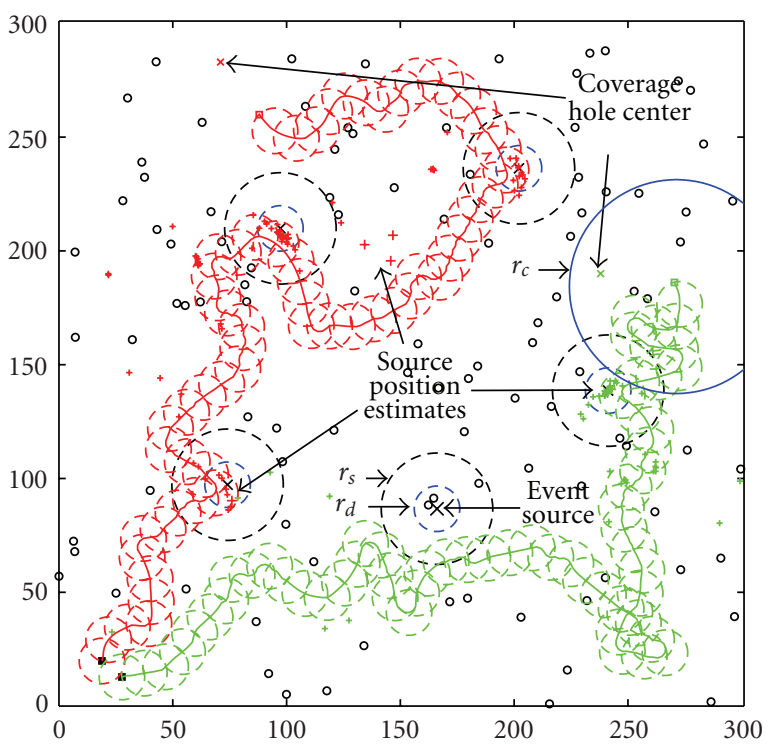

Figure 6: Dynamic distributed path planning using a team of two mobile nodes in the presence of event sources.

navigating towards their current estimated coverage hole positions. Note that in some cases there are sensors within $r_{s}$ from the event sources and these sensors are likely to report the "suspicion" to the passing mobile node. Once a mobile node gets a suspicion message from a static node in its communication range (or its sensor measurement is inside the "suspicion" region, $\tau_{s} \leq z_{i} \leq \tau_{d}$ ), then it switches its target to the estimated location of the event.

The next simulation experiment demonstrates the behavior of the Distributed TS algorithm (with fixed parameters as described above) for sensors fields with different densities (empty, sparse and dense fields). Figure 7 shows the paths followed by three mobile nodes after 300 moving steps. From the figure it is evident that the Distributed TS algorithm is able to easily adapt to different sensor node densities without getting trapped in local minima. Mobile nodes always keep navigating in the sensor field, passing/sampling through uncovered regions and improving coverage. Figure 7(a) shows that in the case of an empty field (no stationary sensors are available) mobile nodes collaborate and navigate similarly to standard search algorithms.

In the next simulation experiment (Figure 8) we investigate the value for the suspicion threshold $\tau_{s}$. Note that there exists a tradeoff in its actual value. If this threshold is set too high, then the mobile will get in the searching mode rarely (clearly $\tau_{s}<\tau_{d}$ ). On the other hand, if this threshold is set too low, then the mobile will be running after frequent false alarms. In this experiment we evaluate the number of detected sources over 20 sensor fields with 100 stationary sensors. In each field 15 nonoverlapping event sources are randomly placed. As shown in Figure 8(a) only a small number of the sources is detected by the stationary sensors (at time zero, about 6.5 sources on average are detected). A group of two mobile sensor nodes using the Distributed TS algorithm is employed. We measure the average number of detected event sources as well as the average coverage

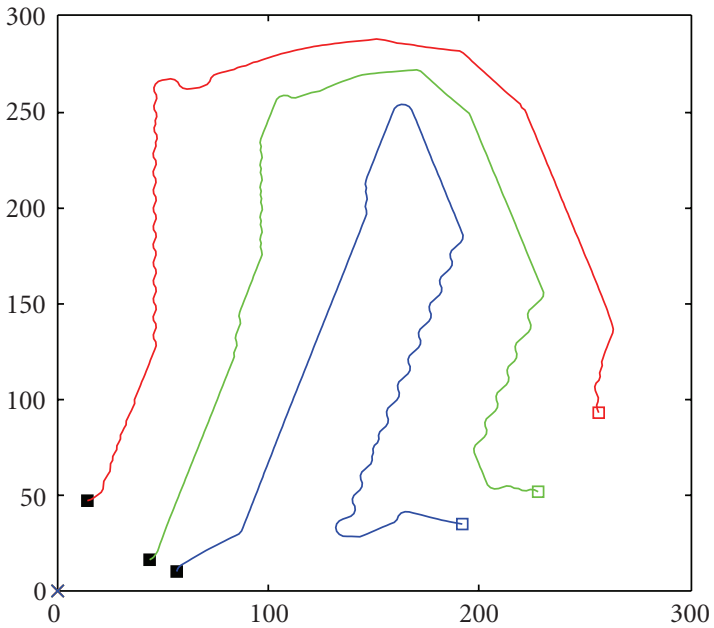

(a) Paths followed in an empty sensor field (0 stationary sensors)

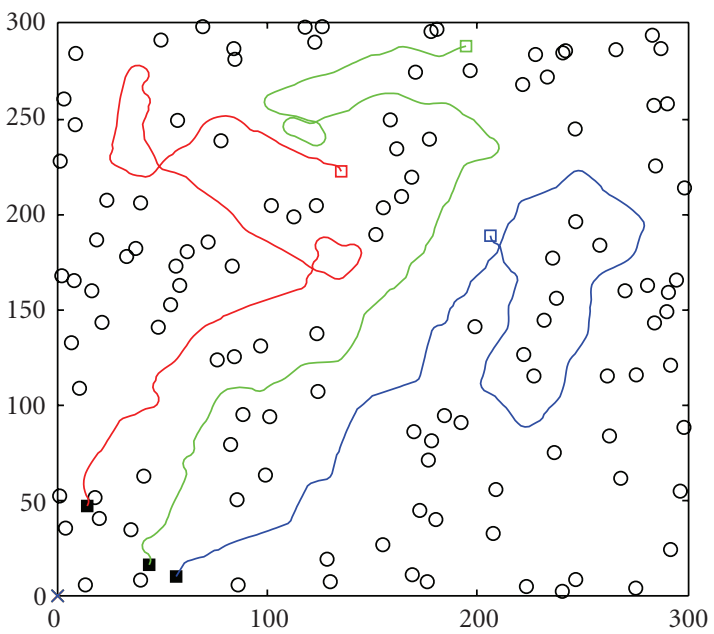

(b) Paths followed in a sparse sensor field (100 stationary sensors)

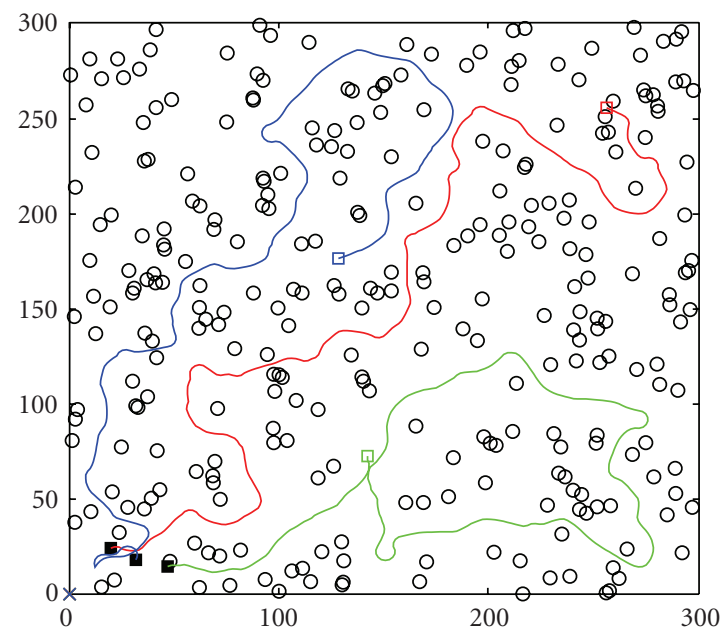

(c) Paths followed in a dense sensor field (300 stationary sensors)

FIGURE 7: Paths followed after 300 moving steps by a set of three mobile sensor nodes using the distributed path planning algorithm for different sensor field densities. 

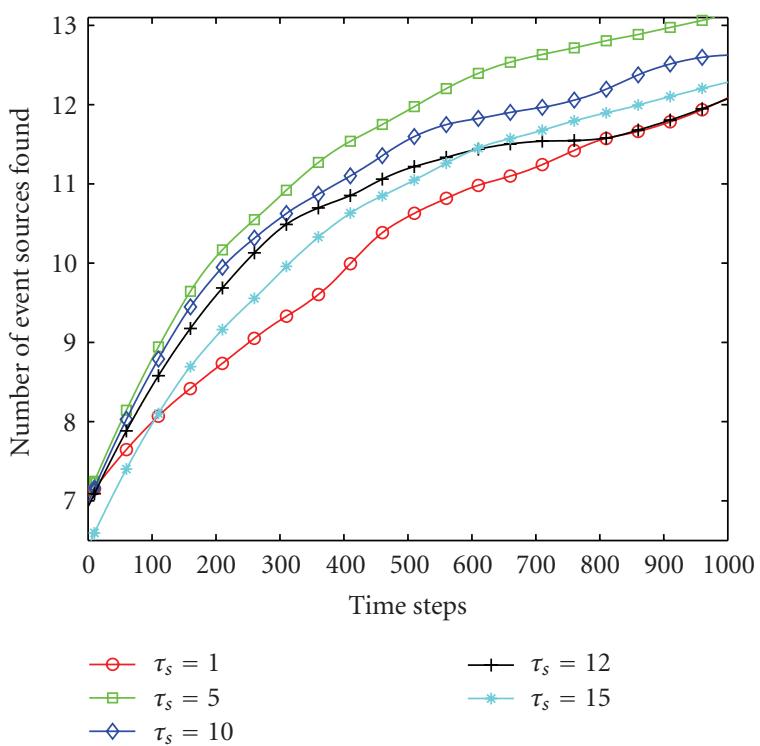

(a) Average number of nonoverlapping event sources found over 20 sensor fields

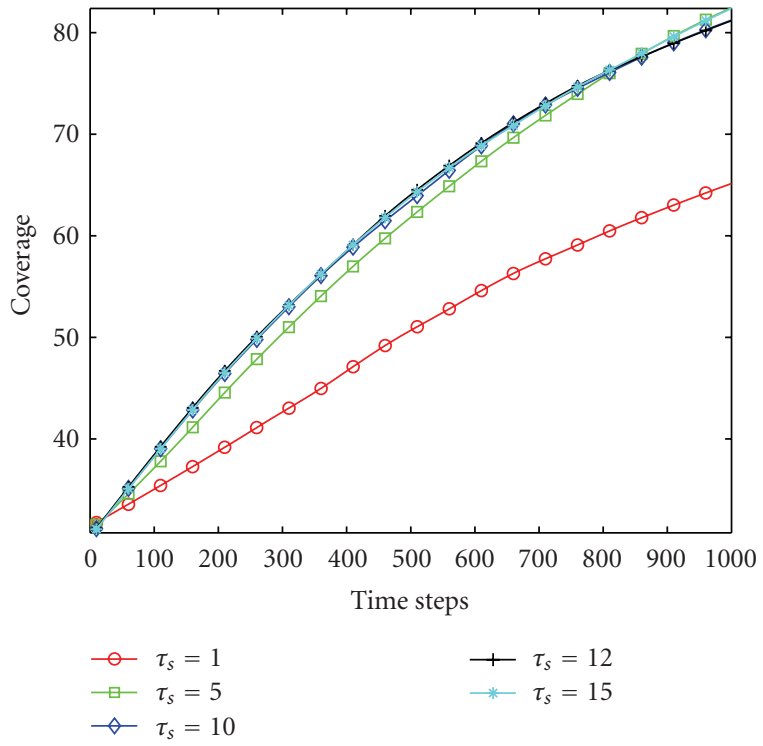

(b) Average coverage improvement over 20 sensor fields

FIGURE 8: Evaluation of the suspicion threshold $\tau_{s}$ optimum value.

improvement for 1000 moving steps. Moreover the following values for other parameters are used: noise variance is $\sigma^{2}=$ $10, \tau_{d}=15, v=5, r_{c}=5 \cdot r_{d}$, and $\ell=r_{c}$.

Figure 8 shows that if the suspicion threshold is set too low $\left(\tau_{s}=1\right)$, then the mobile does run after frequent false alarms and as a result its performance with respect to either the number of detected sources or the area coverage is not very good. As shown in the Figure 8 the best value for this experiment is $\tau_{s}=5$ as this value succeeds coverage close to the maximum which means that it minimizes the uncertainty (or the probability of miss events) and at the same time achieves the maximum rate of detected event sources.

In the next simulation results we compare the following path planning algorithms.
(1) RCM. This algorithm has been developed in $[4,9]$ for cooperative search missions by UAVs. The RCM algorithm uses the cost functions $J_{r}$, $J_{c}$, and $J_{m}$ with the following weights $w_{r}=0.5, w_{c}=0.2, w_{m}=0.3$ and with triangle parameters $\delta=15^{\circ}$ and $\mu=40$. Note that since this algorithm does not use the $J_{t}$ function, it can only navigate in the field to reduce uncertainty (maximize coverage) and cannot move towards a target.

(2) TCM. In this algorithm a central controller decides the next step of each mobile node. Once a mobile node approaches its target destination a new target (coverage hole point) is assign to the mobile using a centralized target assignment scheme where the controller computes the biggest coverage hole in the entire field which is not already assigned to other mobile nodes. The TCM algorithm uses the following cost functions $J_{t}, J_{c}$, and $J_{m}$ with the following weights $w_{t}=0.5, w_{c}=0.2, w_{m}=0.3$ and with parameters $\ell=\sqrt{2 \mathcal{A}}$, where $\mathcal{A}$ is the sensor field area.

(3) TSM. This algorithm is similar to the TCM algorithm (uses a central controller to solve the global target assignment problem). The TSM algorithm uses the following cost functions $J_{t}, J_{s}$, and, $J_{m}$ with the following weights $w_{t}=0.5, w_{s}=0.2$, and $w_{m}=0.3$ and with parameters $\ell=\sqrt{2 \mathcal{A}}$, where $\mathcal{A}$ is the sensor field area.

(4) Distributed TS. As described earlier.

Furthermore the following parameters are used: $r_{d}=10$ $\left(\tau_{d}=15\right), \tau_{s}=5, r_{c}=3 \cdot r_{d}, v=5$ and $\sigma^{2}=10$.

Figure 9 shows the paths followed by two mobile nodes for 500 moving steps when the above algorithms are employed. We use a randomly deployed sensor field with 100 stationary sensor nodes and 4 nonoverlapping event sources. As shown in Figure 9(d) the Distributed TS algorithm achieves better collaboration between the mobiles and detects all the event sources. Better collaboration is achieved because the paths, followed by the mobile sensors using the distributed TS algorithm, have the minimum overlap (almost zero) compared to the other algorithms.

Next we compare the average performance of each algorithm using Monte Carlo simulation. We assume 20 sensor fields with 100 randomly deployed static sensors and 15 static nonoverlapping sources (also placed at random points). Figure 10 is an average over the 20 randomly generated sensor fields and shows that the static sensor network would have detected around 6-7 event-sources on average and the average coverage of the stationary field would be about $30 \%$. Next, a set of two mobile nodes is used for 1000 moving steps. Figure 10 shows that the Distributed TS algorithm outperforms the other algorithms both in the average number of detected event-sources (see (Figure 10(a)), and in the average coverage improvement (Figure 10(b)) and its computation is negligible compared to the RCM algorithm (Figure 10(c)) mainly because there is no 


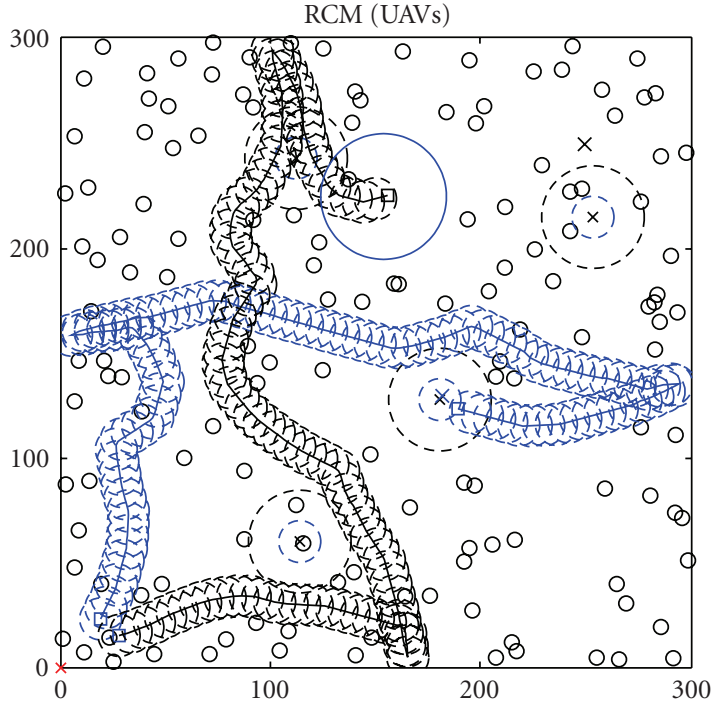

(a) RCM

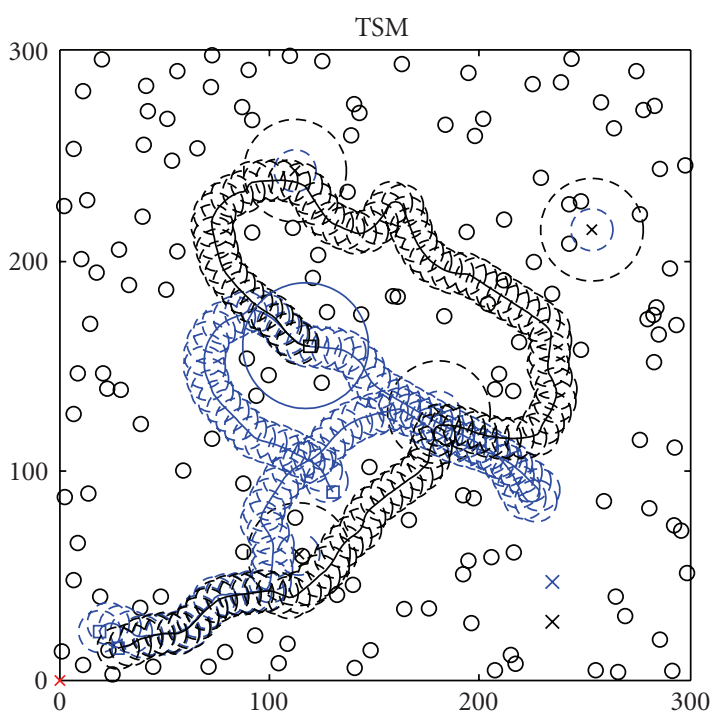

(c) TSM

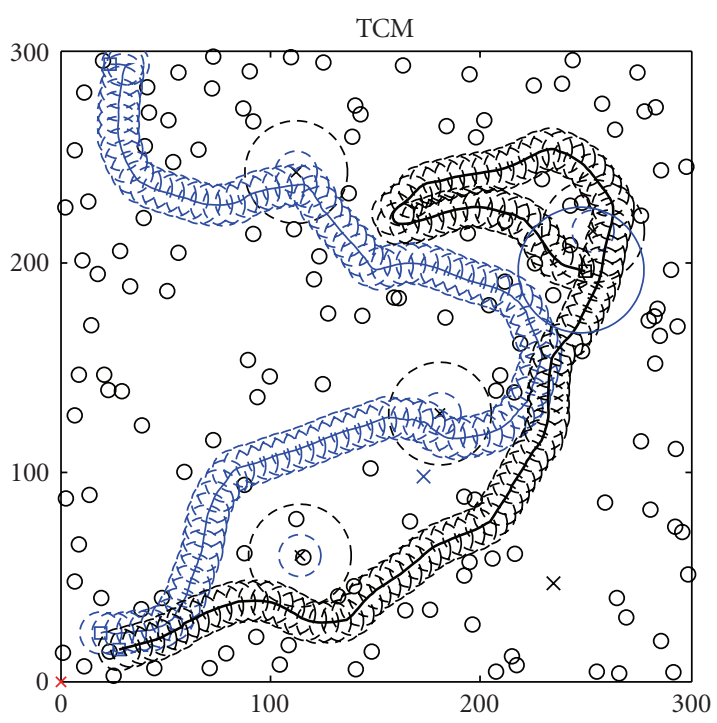

(b) TCM

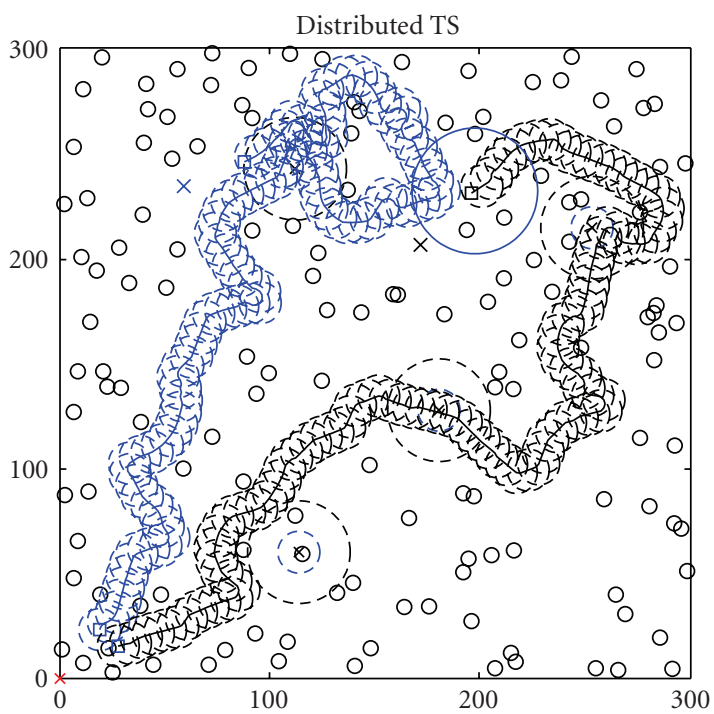

(d) Distributed TS with distributed target assignment

FIGURE 9: Paths followed for 500 moving steps using different path planning algorithms.

need to compute the triangle needed in $J_{r}$. This performances indicates that the Distributed TS algorithm is able to achieve better collaboration between the mobile nodes and its computation efficiency shows that it is a good candidate to be implemented even onto a tiny microcontroller of a mobile sensor node [1].

Finally, as mentioned earlier, fast event detection and area coverage may be two slightly conflicting objectives. Depending on the objective, there may be one or more optimal paths, however, finding them is not easy. Given a path, an easier problem is to determine whether it achieves close to optimal performance. For the coverage objective, this is easily done by observing the coverage overlap between the static and mobile sensors. In that respect, the paths found by the Distributed TS algorithm have performance close to the optimal.

\section{Related Work}

The work presented in this paper is partially related with two research fields, the area coverage in WSNs and path planning in the fields of mobile robotics and UAVs. Although many researchers in the WSNs area have studied the coverage problem, to the best of our knowledge, this is the first time that a general architecture is proposed that combines the coverage problem with distributed path planning algorithms so that the mobile nodes can navigate towards poorly covered areas. The benefit of this approach is that events that would have remained undetected can now be detected.

Next, we present a brief overview of papers that address the coverage problem in the context of WSNs. For a more thorough survey of the coverage problem the reader is referred to $[10,11]$. 


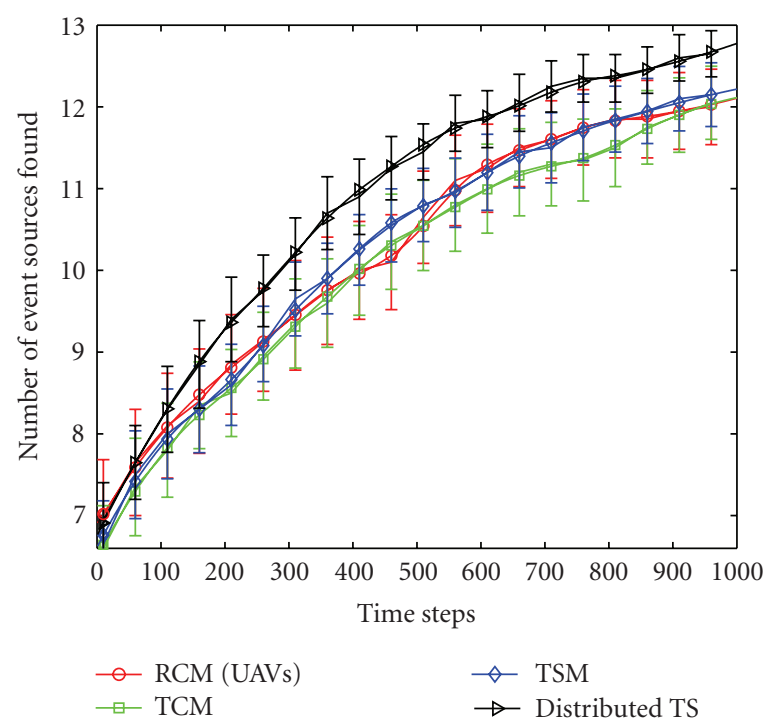

(a) Average number of nonoverlapping event sources found over 20 sensor fields

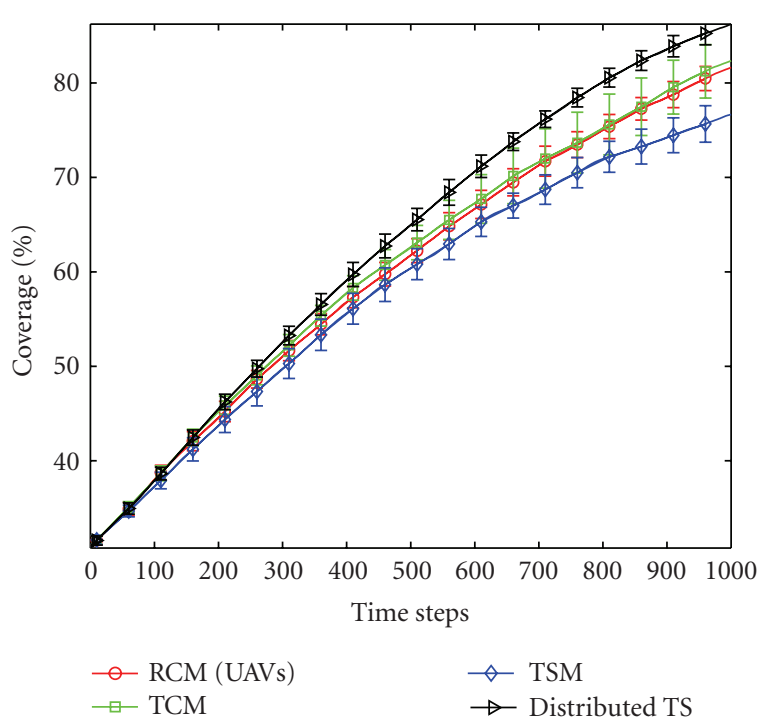

(b) Average coverage improvement over 20 sensor fields

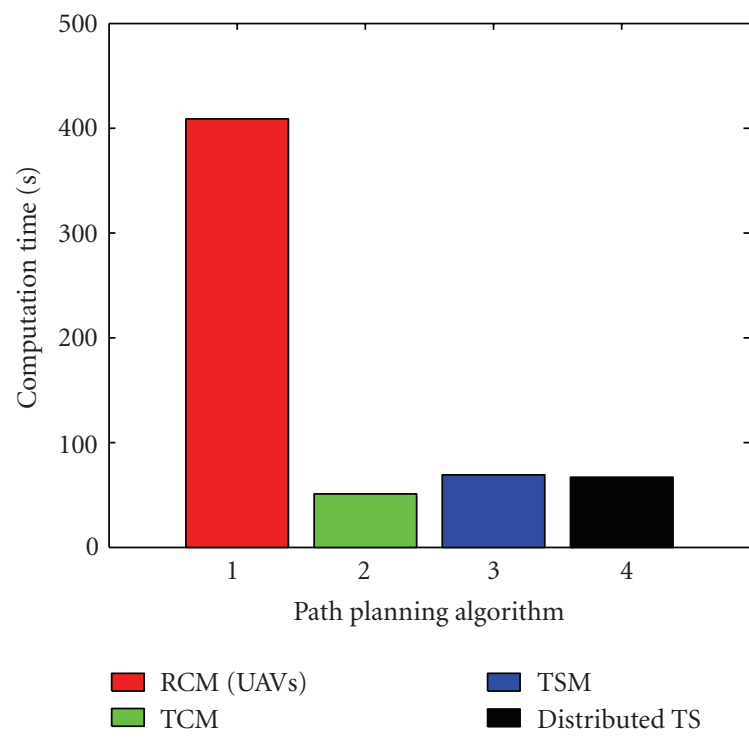

(c) Average computation times

FIGURE 10: Comparison of different path planning algorithms.

In [12] authors proposed the Grid Scan algorithm to find the maximum blind region in order to deploy additional static sensors. The proposed Zoom Algorithm is computationally significantly more efficient than Grid Scan [5]. Next, we present several other approaches that have been proposed in order to determine the coverage holes where mobile nodes can be deployed. All these approaches do not consider the path that the mobile should follow in order to reach to its destination. In [13] authors used Voronoi diagrams to discover the existence of coverage holes. A sensor node compares its sensing disk with the area of its Voronoi polygon to estimate any local coverage hole. Three distributed self-deployment algorithms have been proposed to calculate new optimal positions to which mobile sensors should move to increase coverage: Vector-based (VEC),
Voronoi-based (VOR) and Minimax algorithm. The same authors in [14] describe a bidding protocol for mixed sensor networks that use both static and mobile sensors to achieve a cost balance.

In [15] authors address the problem of enhancing coverage in a mixed sensor network. They present a method to deterministically estimate the exact amount of coverage holes under random deployment using Voronoi diagrams and use the static nodes to estimate the number of additional mobile nodes needed to be deployed and relocated to the holes locations to maximize coverage. In our case we use a small number of mobile nodes that move collaboratively using path planning algorithms in order to enhance the event detection probability of the stationary sensor network. 


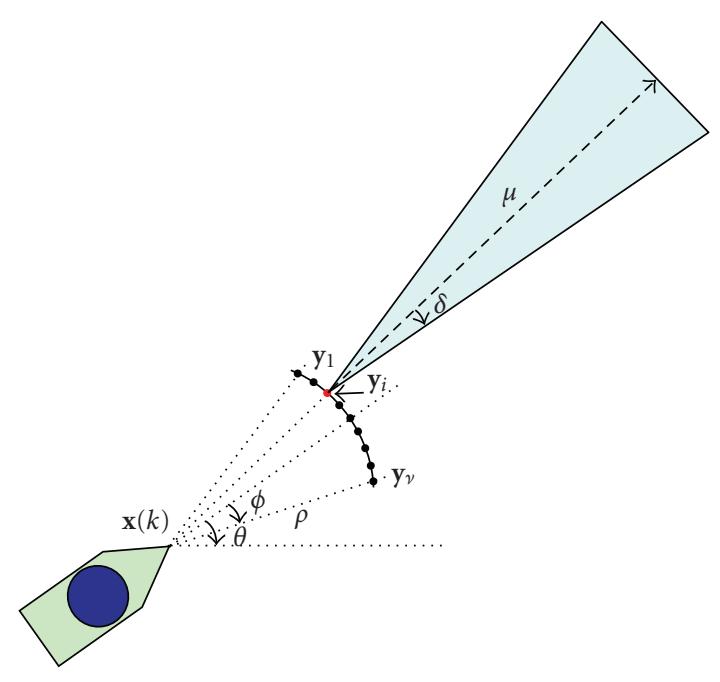

Figure 11: Triangular region used for the $J_{r}(\mathbf{y})$ cost function.

Sensor relocation has been studied in [16], which focuses on finding the target locations of the mobile sensors based on their current locations and the locations of the sensed events. In [17] a polynomial-time algorithm is presented in terms of the number of sensors to determine whether every point in the service area of sensor networks is covered by at least $k$ sensors, where $k$ is a predefined value. In [18], the authors provide a polynomial-time, greedy, iterative algorithm to determine the best placement of one sensor at a time in a grid-based scenario, such that each grid is covered with a minimum confidence level. As already mentioned, none of the aforementioned approaches considers the actual path that each mobile should follow.

Next, we present some path planning algorithms that have been proposed and are relevant to our work. A good overview of motion planning in robotics is given in [19]. As already mentioned, the path planning algorithms presented in this paper have been motivated by the approach in [4] where an approach for cooperative search by a team of distributed agents is presented. In that approach two or more agents move in a geographic environment, cooperatively searching for targets of interest and avoiding obstacles or threats.

Authors in [20] use the concept of Voronoi diagrams and triangulation to provide polynomial-time worst case and best case algorithms for determining maximal breach path and maximal support path, respectively, in a sensing field. On similar lines, in [21], the authors use the concepts of minimal and maximal exposure paths to find out how well an object moving on an arbitrary path can be observed by the sensor network over a period of time. In [22], the authors have focused on the coverage capabilities that result from the continuous random movement of the sensors.

Finally, we present some approaches that address the coverage problem in mobile sensor networks (all sensors are mobile). In [23] authors have looked at the problem of how mobile sensors move collaboratively in order to search a region and also incorporate communication costs into the coverage control problem.

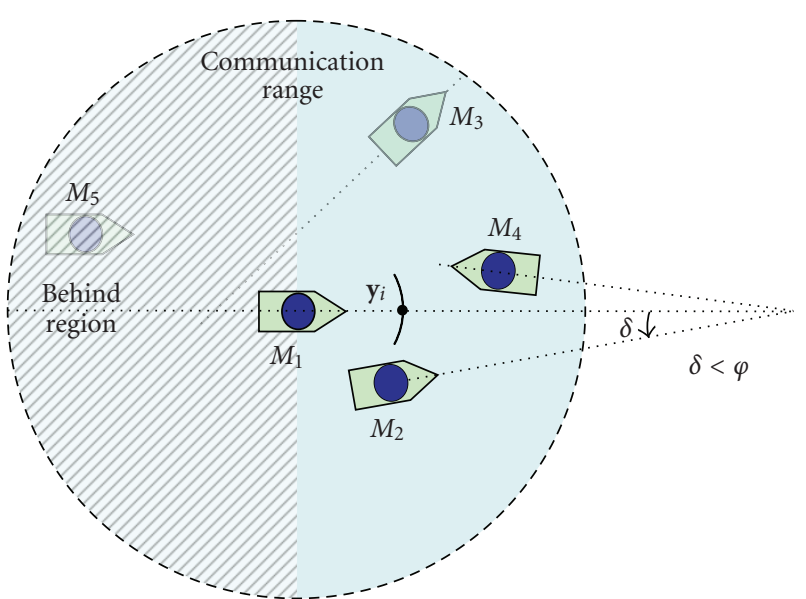

FIGURE 12: Illustration of the set $\Lambda$ for $M_{1}$. Only nodes $M_{2}$ and $M_{4}$ are used in the calculation of $J_{m}\left(y_{i}\right)$.

The coverage concept with regard to the many-robot systems was introduced by Gage [24], who defined three types of coverage: blanket coverage, barrier coverage, and sweep coverage. Potential field techniques for robot motion planning were first described by Khatib [25] and have been widely used in the mobile robotics community for tasks such as local navigation and obstacle avoidance.

Assuming that all sensors have motion capabilities, several approaches have been developed to address the coverage problem using the concept of potential fields [26, 27], and virtual forces in [28]. In a similar fashion, the authors of [27] proposed a potential field-based algorithm in which nodes are treated as virtual particles subjected to virtual force. In [28], the authors presented another virtualforce-based sensor movement strategy to enhance network coverage after an initial random placement of sensors.

\section{Conclusion}

In this paper we propose a collaborate event detection architecture for WSNs consisting of a large number of stationary nodes and a few mobile nodes. The benefit of this architecture is that the mobile nodes collaborate with the stationary nodes so that they sample the areas least covered by the stationary nodes. In this way, events that would have remained undetected can now be detected.

For the proposed architecture we have investigated a family of path planning algorithms that are based on the receding horizon approach. At every step, the mobile controller estimates the cost of moving to a finite set of future positions and moves to the one that achieves the minimum. This cost is a linear combination of certain functions each designed to achieve certain objectives. Five such functions have been investigated in this paper, but more can also be included (e.g., functions that represent obstacles). Among the functions investigated, two had a more local perspective and were designed to avoid stepping to areas covered by immediate neighbors $\left(J_{c}\right.$ and $\left.J_{s}\right)$. The other two were used to give a more global pictures $\left(J_{r}\right.$ and $\left.J_{t}\right)$, and one was explicitly 
used to facilitate the collaboration between the mobiles $\left(J_{m}\right)$. Our investigation yielded the following conclusions with respect to these functions when applied in the context of randomly deployed sensor networks.

(i) $J_{c}$ significantly restricts the movement of mobiles, sometimes it creates fictitious barriers that may "trap" other mobiles, and as a result the simpler $J_{s}$ is able to achieve a better collaboration between the mobile and its neighbors and yield better performance.

(ii) Even though in the context of UAVs $J_{r}$ could achieve very good performance, in the context of randomly deployed sensor networks, its performance was limited and was often outperformed by $J_{t}$. One limitation of the $J_{r}$ function (other than the complexity) is that for reasonably large triangles, due to the random field deployment, the number of sensors that fell in the triangle was fairly constant (proportional to the size and field density) providing no significantly new information.

(iii) When the coverage hole detection algorithm is used to determine the targets of the mobiles, it is usually more beneficial (achieves better collaboration between a group of mobiles) if targets are determined more frequently and closer to the mobile as opposed to more "globally". If a far away target is given to the mobile and is not updated frequently, then it cannot utilize newly discovered information that can help it achieve a better performance.

(iv) When a target is used, the distributed target assignment scheme is more effective in facilitating the collaboration between the mobiles compared to the $J_{m}$ function.

\section{Appendices}

\section{A. Neighboring Sensors Collaboration Cost Function Using the Cognitive Map}

The cost function $J_{c}(\mathbf{y})$, similarly to $J_{s}$, is designed to push the mobile away from areas that have been covered by other sensors (stationary or mobile) using the relevant information from the cognitive map of the mobile node. This function takes a larger value if the candidate position is adequately covered by other sensors and a small value otherwise. Thus, for this paper, the following cost is used:

$$
J_{c}(\mathbf{y})=\frac{1}{\pi r_{d}^{2}} \sum_{\{i, j\} \in D_{l_{d}}(\mathbf{y})} G(i, j),
$$

where $\mathscr{D}_{l_{d}}$ is given by $(5)$ and recall that $\ell_{d}$ is the discretized detection range of the sensor.

\section{B. Cognitive Map Triangular Region Cost Function}

This type of cost function has been proposed in [4] in the context of cooperative control of Unmanned Aerial Vehicles (UAVs) and its main objective is to give an estimate of the future cost (cost-to-go) so that the mobile will avoid local optimal points. This function gives to the path planning algorithm a more global view of the problem but it also requires some global (not just local) information. $J_{r}(\mathbf{y})$ represents the percentage of the covered cells in the cognitive map $G_{k}$ that are included in a triangular region associated with the heading direction of the mobile sensor when going from $\mathbf{x}(k)$ to a point $\mathbf{y}=\mathbf{x}(k+1)$ (see Figure 11). This triangle has two important parameters, the height $\mu$ and the angle $\delta$ :

$$
J_{r}(\mathbf{y})=\frac{1}{\mu^{2} \cdot \tan (\delta)} \sum_{\{i, j\} \in \mathcal{T}(\mathbf{y})} G(i, j),
$$

where $\mathcal{T}(\mathbf{y})$ is the set of all cells $(i, j)$ included in the triangular region associated with the heading direction and the parameters $\mu$ and $\delta$ (see also [29] where these parameters have been investigated in the context of wireless sensor networks). Even though this function has worked very well in the context of searching with UAVs, it does have some limitations in the context of sensor network coverage as will be demonstrated in the simulation results section.

\section{Mobile Sensors Collaboration Cost Function}

In addition we investigated a function proposed in [4] to facilitate the collaboration between mobiles, $J_{m}(\mathbf{y})$ which penalizes each candidate position $\mathbf{y}$ that is close to other mobiles that are heading toward (or returning from) the same direction as the mobile tries to determine its next position. Specifically, when determining its next position, the mobile defines the set $\Lambda$ that includes all other mobiles that are in its communication range and satisfy the following two conditions: (1) the mobiles that do not follow behind and (2) the mobiles that have a heading direction $\delta$ such that $|\theta-\delta| \leq \varphi$ (the two mobiles are heading toward the same direction) or $|\theta-\delta| \geq 180^{\circ}-\varphi$ (the two mobiles are heading toward opposite directions), where $\varphi$ is the maximum allowed difference in heading angle (see Figure 12). The collaboration function is given by

$$
J_{m}(\mathbf{y})=\frac{1}{\beta\|\Lambda\|} \sum_{\lambda \in \Lambda} \beta \exp ^{-r_{\lambda} / 2}
$$

where $\beta$ is a positive design constant and $r_{\lambda}$ is the distance between the candidate position $y$ and the mobile $\lambda$. For more information refer to [4].

\section{Boundaries Cost Function}

To prevent mobiles from stepping outside the field, a boundary cost function $J_{b}(\mathbf{y})$ is introduced that penalizes all 
candidate positions $\mathbf{y}$ that are not included in the field area $\mathcal{A}$. For completeness, the function used is

$$
J_{b}(\mathbf{y})= \begin{cases}1, & \text { if } \mathbf{y} \notin A, \\ 0, & \text { otherwise. }\end{cases}
$$

\section{Acknowledgment}

This work is partly supported by the Cyprus Research Promotion Foundation under contract ENI $\Sigma X / 0505 / 30$.

\section{References}

[1] K. Dantu, M. Rahimi, H. Shah, S. Babel, A. Dhariwal, and G. S. Sukhatme, "Robomote: enabling mobility in sensor networks," in Proceedings of the 4th International Symposium on Information Processing in Sensor Networks (IPSN '05), pp. 404-409, Los Angeles, Calif, USA, April 2005.

[2] D. Estrin, D. Culler, K. Pister, and G. Sukhatme, "Connecting the physical world with pervasive networks," IEEE Pervasive Computing, vol. 1, no. 1, pp. 59-69, 2002.

[3] M. P. Michaelides and C. G. Panayiotou, "Event detection using sensor networks," in Proceedings of the 45th IEEE Conference on Decision and Control (CDC'06), pp. 6784-6789, San Diego, Calif, USA, December 2006.

[4] M. Polycarpou, Y. Yang, Y. Liu, and K. Passino, "Cooperative control design for uninhabited air vehicles," in Cooperative Control: Models, Applications and Algorithms. Vol. I, pp. 283321, Kluwer Academic Publishers, Dordrecht, The Netherlands, 2003.

[5] T. P. Lambrou and C. G. Panayiotou, "Collaborative event detection using mobile and stationary nodes in sensor networks," in Proceedings of the 3rd International Conference on Collaborative Computing: Networking, Applications and Worksharing (CollaborateCom '07), pp. 106-115, New York, NY, USA, November 2007.

[6] A. Dhariwal, G. S. Sukhatme, and A. A. G. Requicha, "Bacterium-inspired robots for environmental monitoring," in Proceedings of IEEE International Conference on Robotics and Automation (ICRA '04), vol. 2, pp. 1436-1443, New Orleans, La, USA, April-May 2004.

[7] T. He, C. Huang, B. M. Blum, J. A. Stankovic, and T. Abdelzaher, "Range-free localization schemes for large scale sensor networks," in Proceedings of the 9th Annual International Conference on Mobile Computing and Networking (MOBICOM '03), pp. 81-95, San Diego, Calif, USA, September 2003.

[8] F. Zhao and L. Guibas, Wireless Sensor Networks: An Information Processing Approach, chapter 2, Morgan Kaufmann, San Francisco, Calif, USA, 2004.

[9] K. Passino, M. Polycarpou, D. Jacques, et al., "Cooperative control for autonomous air vehicles," in Cooperative Control and Optimization, vol. 66, pp. 233-271, Kluwer Academic Publishers, Dordrecht, The Netherlands, 2002.

[10] A. Ghosh and S. K. Das, "Coverage and connectivity issues in wireless sensor networks," in Mobile, Wireless, and Sensor Networks: Technology, Applications, and Future Directions, chapter 9, John Wiley \& Sons, New York, NY, USA, 2006.

[11] M. Cardei and J. Wu, "Coverage in wireless sensor networks," in Handbook of Sensor Networks: Compact Wireless and Wired Sensing Systems, chapter 19, CRC Press, Boca Raton, Fla, USA, 2004.
[12] X. Shen, J. Chen, and Y. Sun, "Grid scan: a simple and effective approach for coverage issue in wireless sensor networks," in Proceedings of IEEE International Conference on Communications (ICC '06), vol. 8, pp. 3480-3484, Istanbul, Turkey, July 2006.

[13] G. Wang, G. Cao, and T. La Porta, "Movement-assisted sensor deployment," in Proceedings of the 23rd Annual Joint Conference of the IEEE Computer and Communications Societies (INFOCOM '04), pp. 1-11, Hong Kong, March 2004.

[14] G. Wang, G. Cao, and T. La Porta, "A bidding protocol for deploying mobile sensors," in Proceedings of the 11th IEEE International Conference on Network Protocols (ICNP '03), pp. 315-324, Atlanta, Ga, USA, November 2003.

[15] A. Ghosh, "Estimating coverage holes and enhancing coverage in mixed sensor networks," in Proceedings of the 29th Annual IEEE International Conference on Local Computer Networks (LCN'04), pp. 68-76, Tampa, Fla, USA, November 2004.

[16] Z. Butler and D. Rus, "Event-based motion control for mobilesensor networks," IEEE Pervasive Computing, vol. 2, no. 4, pp. 34-42, 2003.

[17] C.-F. Huang and Y.-C. Tseng, "The coverage problem in a wireless sensor network," in Proceedings of the 2nd ACM International Workshop on Wireless Sensor Networks and Applications (WSNA '03), pp. 115-121, ACM Press, San Diego, Calif, USA, September 2003.

[18] S. S. Dhillon, K. Chakrabarty, and S. S. Iyengar, "Sensor placement for grid coverage under imprecise detections," in Proceedings of the 5th International Conference on Information Fusion (FUSION '02), vol. 2, pp. 1581-1587, Annapolis, Md, USA, July 2002.

[19] J.-C. Latombe, Robot Motion Planning, Kluwer Academic Publishers, New York, NY, USA, 1992.

[20] S. Meguerdichian, F. Koushanfar, M. Potkonjak, and M. B. Srivastava, "Coverage problems in wireless ad-hoc sensor networks," in Proceedings of 20th Annual Joint Conference of the IEEE Computer and Communications Societies (INFOCOM '01), vol. 3, pp. 1380-1387, Anchorage, Alaska, USA, April 2001.

[21] S. Meguerdichian, F. Koushanfar, G. Qu, and M. Potkonjak, "Exposure in wireless ad-hoc sensor networks," in Proceedings of the 7th Annual International Conference on Mobile Computing and Networking (MOBICOM '01), pp. 139-150, ACM Press, Rome, Italy, July 2001.

[22] B. Liu, P. Brass, O. Dousse, P. Nain, and D. Towsley, "Mobility improves coverage of sensor networks," in Proceedings of the 6th ACM International Symposium on Mobile Ad Hoc Networking and Computing (MOBIHOC '05), pp. 300-308, Urbana-Champaign, Ill, USA, May 2005.

[23] W. Li and C. G. Cassandras, "Distributed cooperative coverage control of sensor networks," in Proceedings of the 44th IEEE Conference on Decision and Control, and the European Control Conference (CDC-ECC '05), pp. 2542-2547, Seville, Spain, December 2005.

[24] D. W. Gage, "Command control for many-robot systems," in Proceedings of the 19th Annual AUVS Technical Symposium, pp. 22-24, Huntsville, Ala, USA, June 1992.

[25] O. Khatib, "Real-time obstacle avoidance for manipulators and mobile robots," International Journal of Robotics Research, vol. 5, no. 1, pp. 90-98, 1986.

[26] S. Poduri and G. S. Sukhatme, "Constrained coverage for mobile sensor networks," in Proceedings of IEEE International Conference on Robotics and Automation (ICRA '04), vol. 1, pp. 165-171, New Orleans, La, USA, April-May 2004. 
[27] A. Howard, M. Mataric, and G. Sukhatme, "Mobile sensor network deployment using potential fields: a distributed, scalable solution to the area coverage problem," in Proceedings of the 6th International Symposium on Distributed Autonomous Robotic Systems (DARS '02), pp. 299-308, Fukuoka, Japan, June 2002.

[28] Y. Zou and K. Chakrabarty, "Sensor deployment and target localization based on virtual forces," in Proceedings of the 22nd Annual Joint Conference on the IEEE Computer and Communications Societies (INFOCOM '03), vol. 2, pp. 12931303, San Francisco, Calif, USA, March-April 2003.

[29] T. P. Lambrou and C. G. Panayiotou, "Improving area coverage using mobility in sensor networks," in Proceedings of the International Conference on Intelligent Systems And Computing: Theory And Applications (ISYC '06), pp. 1-8, Ayia Napa, Cyprus, July 2006. 